\title{
Los polinomios particulares: Una definición para exploraciones cartesianas
}

\author{
Jorge E. Mendoza G. \\ jorge.mendoza@correounivalle.edu.co \\ Instituto de Educación y Pedagogía \\ Universidad del Valle-Cali, Colombia
}

Recibido: 6 Marzo 2013 Aceptado: 12 Junio 2013

Resumen. El objetivo principal de este trabajo es presentar formalmente dos definiciones que permiten hallar relaciones geométricas y algebraicas a través de la adición y sustracción de una función y su derivada, lo que permite explorar sus gráficos de nuevas maneras. Como consecuencia de estas definiciones, se presenta el caso particular de las funciones polinómicas, donde se obtiene como resultado principal un curioso teorema acerca de parábolas obtenidas a través de las definiciones dadas.

Palabras clave: Polinomios particulares (P.P.), series de potencias, derivada.

Abstract. This paper formally presents two definitions that allow to find geometric and algebraic relations through the addition and subtraction of a function and its derivative, thus enabling new ways of exploring their graphics. As a consequence of these definitions, the particular case of polynomial functions is presented, where a curious theorem about parabols is obtained as a main result.

KeyWords: Particular Polynomials (P.P.), power series, derivative.

\subsection{Introducción}

Históricamente, uno de los precursores del uso de la representación de funciones mediante series de potencias fue Isaac Newton con el descubrimiento de la serie binomial entre 1664-1665. A partir de este desarrollo, existió la posibilidad de obtener múltiples representaciones para funciones trascendentes, de potencias negativas como $(1+x)^{-1}$, etc. En este trabajo se consideran series de potencias de la 
forma $f(x)=\sum_{n=0}^{\infty} a_{n} x^{n}$ y se define lo que es un polinomio particular (P.P.), utilizando elementos como la función misma y su derivada, denotada por $\frac{d f}{d x}$.

\section{2 ¿De dónde surgió el problema?}

El siglo XVII fue un gran catalizador de los resultados y métodos más prolíficos en las matemáticas iniciadas por Newton y Leibniz; una de estas herramientas fueron las técnicas usadas al aplicar las series infinitas. A continuación se presenta una forma alternativa de combinar funciones y sus derivadas, en donde se combinan series finitas e infinitas de potencias. Con esas combinaciones es posible obtener resultados atractivos que producen nuevas comprensiones sobre conceptos ya conocidos.

En el libro "The historical Development of the calculus" de C.H Edwards, Jr, aparece la suma $f(x)=$ $\sum_{n=0}^{\infty} a_{n} x^{n}$. Está representación en series de potencias presenta curiosas relaciones con diferentes campos de las matemáticas, especialmente en el cálculo infinitesimal. Al tener el desarrollo en potencias, se puede pensar en derivar la expresión término a término, obteniendo $f^{\prime}(x)=\sum_{n=1}^{\infty} n a_{n} x^{n-1}$. De esta forma escudriñando un poco $f(x)$ y $f^{\prime}(x)$ se considera la suma y la resta de ambas series de potencias, lo que permite dar una definición general que al menos se sabe que va a ser útil en los casos en que la función y la derivada se pueden expandir en series de potencias convergentes:

$$
f(x)+f^{\prime}(x) \text { y } f(x)-f^{\prime}(x)
$$

Este artificio se realiza para luego definir dos operadores que producen los dos polinomios particulares para cada función $f$. Dichos operadores se simbolizan por $\tau_{+} \mathrm{y} \tau_{-}, \mathrm{y}$ los dos polinomios particulares por $\tau_{+}(f(x))$ y $\tau_{-}(f(x))$ En un nivel más profundo, el problema surge a partir de la curiosidad del autor por indagar las relaciones entre una función y su derivada, tanto algebraica como gráficamente.

\subsection{Dos definiciones usando series de potencias}

Se pueden tomar los polinomios que tienen un número finito de términos como un caso particular de las series de potencias infinitas, pero en las que de cierto grado en adelante todos los coeficientes siguientes son ceros. Defínase los PP en particular para las funciones polinómicas así: 


\section{Definición 1.1}

Sea $f(x)=a_{0}+a_{1} x+a_{2} x^{2}+a_{3} x^{3}+\cdots+a_{n} x^{n}$ un polinomio de grado $n$ donde $n \in N$, se define el "polinomio particular para la suma en $f(x)^{\prime \prime}$ como

$$
\begin{aligned}
& \tau_{+}(f(x))=\mathbf{f}(\mathbf{x})+\frac{\mathbf{d f}}{\mathbf{d x}}=\left(a_{0}+a_{1} x+a_{2} x^{2}+a_{3} x^{3}+\cdots a_{n} x^{n}\right) \\
& +\left(a_{1}+2 a_{2} x+3 a_{3} x^{2}+\ldots+n a_{n} x^{n-1}\right)
\end{aligned}
$$

\section{Definición 1.2}

Sea $f(x)=a_{0}+a_{1} x+a_{2} x^{2}+a_{3} x^{3}+\cdots+a_{n} x^{n}$ un polinomio de grado $n$ donde $n \in N$, se define el "polinomio particular para la resta en $f(x)$ " como

$$
\begin{aligned}
\tau_{-}(f(x))=f(x)-\frac{d f}{d x}= & \left(a_{0}+a_{1} x+a_{2} x^{2}+a_{3} x^{3}+\cdots+a_{n} x^{n}\right) \\
- & \left(a_{1}+2 a_{2} x+3 a_{3} x^{2}+\ldots+n a_{n} x^{n-1}\right)
\end{aligned}
$$

\subsection{Un caso particular que conduce a un Teorema}

A partir de las definiciones anteriores, si $f(x)$ es una función polinómica, se pueden determinar $\tau_{+}(f(x))$ y $\tau_{-}(f(x))$ como polinomios finitos específicos en donde no figuran ya explícitamente las derivadas de la función inicial. Así, por ejemplo para $f(x)=\sum_{n=0}^{2} a_{n} x^{n}=a_{0}+a_{1} x+a_{2} x^{2}$

$$
f(x)=a_{0}+a_{1} x+a_{2} x^{2}
$$

sus P.P. correspondientes están dados por

$$
\begin{aligned}
& \tau_{+}(f(x))=\left(a_{0}+a_{1} x+a_{2} x^{2}\right)+\left(a_{1}+2 a_{2} x\right)=\left(a_{0}+a_{1}\right)+x\left(a_{1}+2 a_{2}\right)+a_{2} x^{2} \\
& \tau_{-}(f(x))=\left(a_{0}+a_{1} x+a_{2} x^{2}\right)-\left(a_{1}+2 a_{2} x\right)=\left(a_{0}-a_{1}\right)+x\left(a_{1}-2 a_{2}\right)+a_{2} x^{2}
\end{aligned}
$$

A partir de los P.P. para la suma y resta se establece el siguiente "teorema" como una consecuencia de las definiciones 1.3 y 1.3 . 


\section{Teorema 1.1}

Sea $f(x)=a_{0}+a_{1} x+a_{2} x^{2}$ con P.P. $\tau_{+}(f(x))$ y $\tau_{-}(f(x))$, entonces es posible encontrar dos parábolas $\tau_{+}(f(x))$ y $\tau_{-}(f(x))$ cuyo punto de corte coincida exactamente con el vértice de la gráfica cartesiana de $f(x)$ y la distancia del vértice de $f(x)$ al vértice de $\tau_{+}(f(x))$ es igual a la distancia del mismo a $\tau_{-}(f(x))$.

Demostración. Dado que $f(x)=a_{0}+a_{1} x+a_{2} x^{2}$, entonces por definición, tiene P.P. $\tau_{+}(f(x))$ y $\tau_{-}(f(x))$. El vértice de $f$ es el punto

$$
V_{1}\left(\frac{-a_{1}}{2 a_{2}}, a_{0}-\frac{a_{1}^{2}}{4 a_{2}}\right)
$$

De esta manera, al operar $f(x)$ con $\tau_{+}(f(x))$ y $\tau_{-}(f(x))$ se obtiene

$$
\begin{aligned}
& \tau_{+}(f(x))=\left(a_{0}+a_{1}\right)+x\left(a_{1}+2 a_{2}\right)+a_{2} x^{2} \\
& \tau_{-}(f(x))=\left(a_{0}-a_{1}\right)+x\left(a_{1}-2 a_{2}\right)+a_{2} x^{2}
\end{aligned}
$$

De aquí, las tres parábolas tienen un punto de intersección común, considérese $x=\frac{-a_{1}}{2 a_{2}}$, de esta forma evaluando en $\tau_{+}(f(x))$ y $\tau_{-}(f(x))$ se obtiene

$$
\begin{aligned}
& \tau_{+} f\left(\frac{-a_{1}}{2 a_{2}}\right)=a_{0}-\frac{a_{1}^{2}}{4 a_{2}} \\
& \tau_{-} f\left(\frac{-a_{1}}{2 a_{2}}\right)=a_{0}-\frac{a_{1}^{2}}{4 a_{2}},
\end{aligned}
$$

que es el valor obtenido en el vértice $V_{1}$; por lo tanto las dos parábolas $\tau_{+}(f(x))$ y $\tau_{-}(f(x))$ tienen como punto común el vértice de la función $f$

La demostración de la otra parte del teorema: "la distancia del vértice de $f(x)$ al vértice de $\tau_{+}(f(x))$ es igual a la distancia del mismo a $\tau_{-}(f(x))^{\prime \prime}$, se deja como ejercicio al lector.

Sugerencia: Se puede utilizar, entre otras estrategias, la fórmula usual de la distancia entre dos puntos $d(A, B)$ insertando los vértices de las dos parábolas $V_{1}$ y $V_{2}$.

\subsection{Conclusiones}

Es claro que la forma de resolver el problema y buscar parábolas simétricas que pasen por el vértice de una parábola dada parece artificial e indirecta, en el sentido de que es necesario tener bajo la manga las definiciones 1 y 2; pero este sencillo artificio de asociar dos polinomios o dos series de potencias a cada función y su derivada resulta una fuente muy prolífica para generar problemas algebraicos y gráficos para muchos tipos de curvas y sus puntos privilegiados. 


\section{Bibliografía}

[1] Apostol, T. (2001). Calculus (Vol. 1). Barcelona: Editorial Reverté.

[2] Edwards, B. (1999). Cálculo y Geometría Analítica (Vol. Sexta Edición). (L. A. Rapún, Trad.) Madrid: McGraw-Hill.

[3] Edwards, C. J. (1937). The Arithmetic of the Infinite. In C. E. Jr., The Historical Development of the Calculus (p. 166). New York: Springer-Verlag.

[4] Guicciardini, N. (2003). A history of analysis. En H. N. Jahnke (Ed.), A History of Analysis (History of Mathematics, vol. 24). Princeton, RI: American Mathematical Society-London Mathematical Society. 